\title{
Core-Collapse Supernova Simulations with CHIMERA
}

O.E. Bronson Messer ${ }^{\star a} \dagger \dagger$, Stephen W. Bruenn ${ }^{d}$, John Blondin ${ }^{e}$, Merek A. Chertkow ${ }^{b}$, W. Raphael Hix ${ }^{c b}$, Ching-Tsai Lee ${ }^{b}$, Eric J. Lentz ${ }^{b c}$, Pedro Marronetti ${ }^{d}$, Anthony Mezzacappa $^{c b}$, and Konstantin N. Yakunin ${ }^{d}$

${ }^{a}$ Leadership Computing Facility, Oak Ridge National Laboratory Oak Ridge, TN 37831

${ }^{b}$ Department of Physics \& Astronomy, University of Tennessee Knoxville, TN 37996

${ }^{c}$ Physics Division, Oak Ridge National Laboratory Oak Ridge, TN 37831

${ }^{d}$ Department of Physics, Florida Atlantic University Boca Raton, FL 33431

${ }^{c}$ Department of Physics, North Carolina State University Raleigh, NC 27695

E-mail: bronsoneornl.gov

\begin{abstract}
Unraveling the core-collapse supernovae mechanism is an outstanding computational challenge and the problem remains essentially unsolved despite more than four decades of effort. However, progress in realistic modeling has occurred recently through the availability of petascale platforms and the increasing sophistication of supernova codes. CHIMERA is a code we have developed to simulate core-collapse supernovae in one, two, and three spatial dimensions, incorporating modules for ray-by-ray neutrino transport and nuclear kinetics. In addition to this base functionality, CHIMERA includes several other features designed to provide additional capability. For example, the availability of Lagrangian tracer particles in CHIMERA allows us to produce realistic, post-processed estimates for a variety of multi-messenger observables, including supernova nucleosynthesis and gravitational wave signatures.
\end{abstract}

11th Symposium on Nuclei in the Cosmos, NIC XI

July 19-23, 2010

Heidelberg, Germany

\footnotetext{
*Speaker.

$\dagger$ This work was supported by the NSF under grants OCI-0749242, PHY-0855315, and AST-0653376; by NASA under grant 07-ATFP07-0011; and by the Office of Nuclear Physics and Office of Advaced Scientific Computing Research, U. S. Department of Energy. The work was performed at the Oak Ridge National Laboratory, which is managed by UT-Battelle, LLC under Contract No. DE-AC05-00OR22725. This research used resources of the Oak Ridge Leadership Computing Facility at the Oak Ridge National Laboratory provided through the INCITE program. This research was supported in part by the National Science Foundation through TeraGrid resources provided by the National Institute for Computational Sciences under grant number TG-MCA08X010.
} 


\section{The Complexity of Core-Collapse Supernova Modeling}

How the stalled core-collapse supernova shock is revived and proceeds to disrupt the massive star that serves as its progenitor is the central (unsolved) question of supernova theory. The specifics of this process formed the basis of many of the first simulations ever performed in computational astrophysics [1], and have been pursued on supercomputers ever since. Although realistic modeling of the core-collapse supernova mechanism is a daunting computational challenge, examination of some of the known physical processes in the event does provide a short list of probable actors. "Ray-by-ray" simulations [2-5] capture much of the neutrino transport realism in multidimensional models, as contributions from lateral neutrino transport, which will likely only be important below the neutrinospheres in the proto-neutron star, can be included with the addition of a neutrino advection scheme. The addition of a prescription for nuclear burning to a multidimensional ray-by-ray radiation hydrodynamics scheme allows much of the known physics in the problem to be competently addressed. CHIMERA—our core-collapse supernova code-has been developed with these notions in mind. The simulation results provide reasonable physical fidelity (i.e., enough to determine the nature and many of the consequences of the explosion mechanism) at a tractable cost.

\section{CHIMERA \& Nuclear Kinetics}

CHIMERA can well be described as a "chimera" of three, separate, rather mature codes. The codes are tightly coupled in a single executable through a set of interface routines. The primary code modules are designed to evolve the stellar gas hydrodynamics (VH1), the "ray-by-rayplus" neutrino transport (MGFLD-TRANS), and the thermonuclear kinetics (XNET). These three "heads" are augmented by a sophisticated equation of state for nuclear matter (e.g. LS-EOS [6]) and a self-gravity solver capable of an approximation to general-relativistic gravity. The general features of two-dimensional simulations using CHIMERA have been reported in Bruenn et al. [7].

Ideally, neutrino transport would be implemented with full multidimensional Boltzmann transport. As a compromise between accuracy and computational intensity, we employ a "ray-by-rayplus" approximation (cf. [4]) for neutrino transport, whereby the lateral effects of neutrinos such as lateral pressure gradients (in optically thick conditions), neutrino advection, and velocity corrections are taken into account, but transport is performed only in the radial direction. The neutrino opacities employed for the simulations are the "standard" ones described in [8], with the isoenergetic scattering of nucleons replaced by the more complete formalism of [9], which includes nucleon blocking, recoil, and relativistic effects, and with the addition of nucleon-nucleon bremsstrahlung [10] with the kernel reduced by a factor of five in accordance with the results of [11].

The equation of state (EOS) of [6] is currently employed for matter at high densities. For regions not in NSE, an EOS with a nuclear component consisting of 14 alpha-particle nuclei $\left({ }^{4} \mathrm{He}\right.$ to ${ }^{60} \mathrm{Zn}$ ), protons, neutrons, and an ironlike nucleus is used. An electron-positron EOS with arbitrary degeneracy and degree of relativity spans the entire density-temperature regime of interest. The nuclear composition in the non-NSE regions is evolved by the thermonuclear reaction network of [12]. This is a fully implicit general purpose reaction network; however, currently we have 


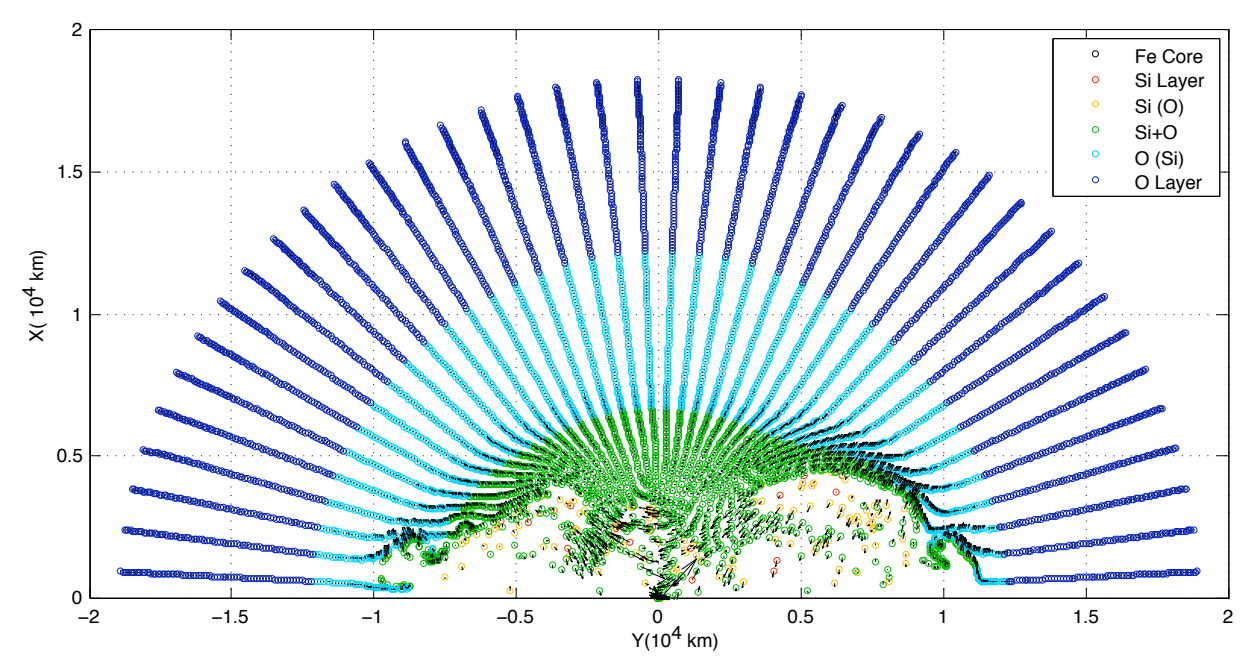

Figure 1: Tracer particle snapshot from 1s post-bounce in a CHIMERA simulation. Particles are colorcoded according to their original locations in the progenitor compositional profile. The progenitor star is the $25 \mathrm{M}_{\odot}$ model of Heger et al. [15]

implemented only a so-called $\alpha$-network, i.e. only reactions linking the 14 alpha nuclei from ${ }^{4} \mathrm{He}$ to ${ }^{60} \mathrm{Zn}$ are used. Because the $\alpha$-network neglects reaction flows involving neutron-rich nuclei, it provides only estimates of the energy generation rates for nuclear burning stages encountered in the supernova [e.g., $\pm 50 \%$ for oxygen burning; 13]. Detailed nucleosynthesis requires evolving 150 or more nuclear isotopes throughout the matter which is ultimately ejected. Simple replacement of the 14 element network with 150 isotopes in the fully-implicit backward-Euler integration scheme represents a several hundred-fold increase in the computational cost. Given the current $\approx 5 \%$ of execution time required by the alpha network in the current simulations, this cost is prohibitive if implemented naïvely. However, to better confront observations of isotopic abundances in the event, we have also finished the initial development of a tracer particle tracking module in CHIMERA to allow more detailed post-processing nucleosynthesis calculations to be performed [14].

\section{Tracer Particles}

The hydrodynamic scheme in CHIMERA is Eulerian, wherein the state of the stellar material is defined on a grid that is fixed in space. However, as individual parcels of stellar material encounter various thermodynamic conditions as they wind their way through the outer and inner core of the star, the composition of these parcels will be strongly modified, most notably by significant temperature differences. The value of Lagrangian tracer particles in an Eulerian hydrodynamic scheme is the ability to follow the evolution of mass elements from start to finish. This can be used, for example, to compute post-processing nucleosynthesis with a much larger thermonuclear reaction network (see Fig. 2 as an example), or map the final location of progenitor compositional features (see Fig. 1). The tracers in CHIMERA are implemented with a simple Euler scheme, implicitly assuming the velocity remains constant over a timestep. With the small timestep imposed by the Courant condition on the spherical polar grid, the Euler scheme proves entirely consistent with more complex schemes like the predictor-corrector, and has a lower data overhead. This lower 


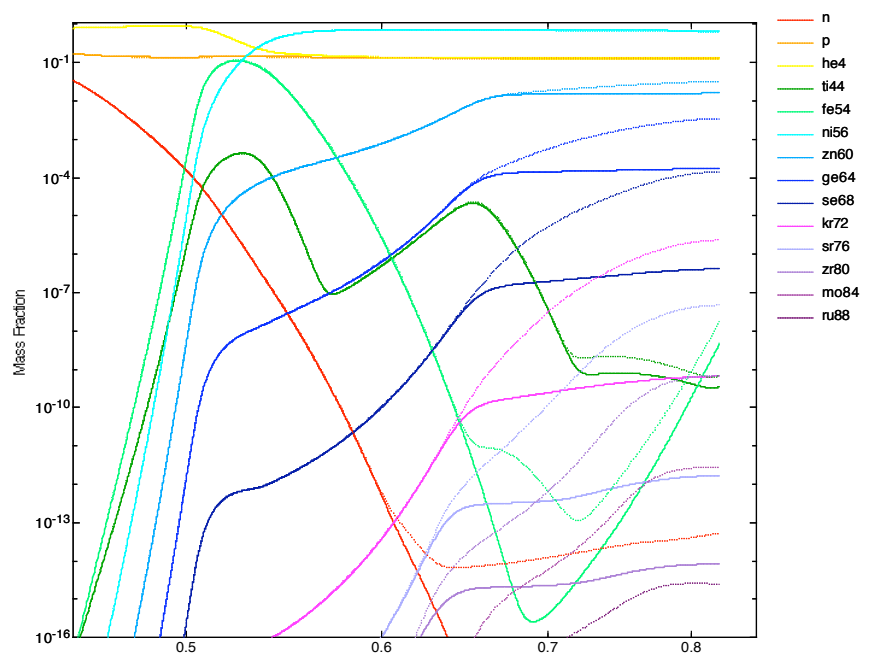

Figure 2: $v p$-process nucleosynthesis calculation for a tracer particle trajectory from an explosion simulation using the $12 \mathrm{M}_{\odot}$ progenitor of Heger et al. [15]. Shown are evolutions with neutrino-nucleus reactions included (dotted) and ignored (solid). The ability to use larger and more complete nuclear networks to determine compositional evolution is the primary advantage of post-processing particle data from CHIMERA.
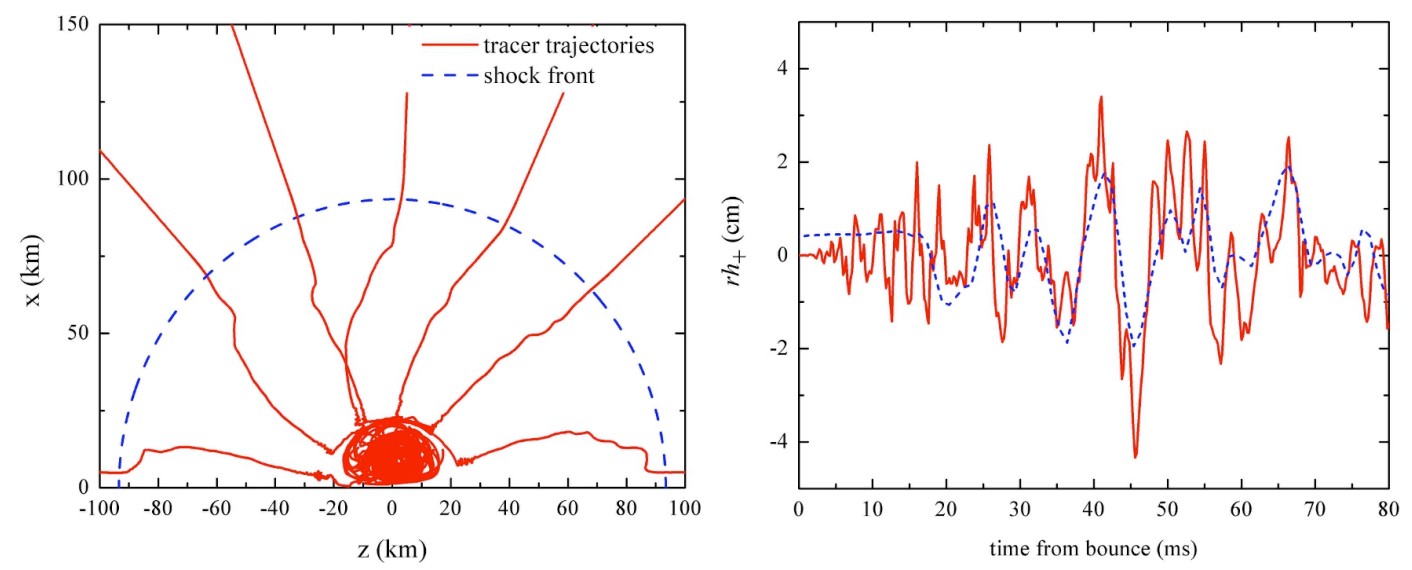

Figure 3: Left: Deflections of infalling tracer particles passing through the shock at $60 \mathrm{~ms}$ post-bounce in a $15 \mathrm{M}_{\odot}[15]$ simulation. The shock front position shown is angle-averaged. Right: Comparison between the matter signal (solid red) and signal calculated using the tracers (dashed blue).

overhead is important, because the unique parallel decomposition of the CHIMERA code poses an additional challenge. CHIMERA uses a global stripe-wise decomposition between processors, as opposed to block decomposition. This requires a transpose of the data between directional sweeps, but obviates coordination of neighboring blocks and simplifies coupling with the ray-byray neutrino transport modules in CHIMERA. This simplicity for the transport, however, made implementing the tracers much more challenging. Instead of tracers occasionally transiting from one block to a neighbor, the global transform in VH-1 requires accounting for such a transit with each directional sweep. This strip-wise decomposition also requires directional splitting of the tracer evolution. Our testing has shown that such splitting, which is actually more consistent with the hydrodynamic evolution, has negligible effect on the tracer evolution. 
In addition to their originally-intended purpose as matter tracers for post-processed nucleosynthesis calculations, we have recently used the Lagrangian tracers to confirm our understanding of generation mechanisms for gravitational waves $(\mathrm{GW})$ in CHIMERA simulations [16]. In particular, using data from the tracer particles, Yakunin et al. [16] were able to show that the low-frequency component of the prompt (i.e. from core bounce to roughly 50-75 ms post bounce) GW signal in CHIMERA simulations stems from matter deflections at the supernova shock (see Fig. 3). In general, the ability to follow matter parcel trajectories allows us to follow and quantify a number of separate physical processes in supernova simulations. The possession of a tool to tease apart the multifarious feedbacks present in the simulations contributes appreciably to the insight that can be gleaned through computation. This is an important capability, as the ability to simultaneously confront observations of the myriad of multi-messenger signals from core-collapse supernovae is the preeminent measure of the value of our multiphysics simulations.

\section{References}

[1] S. A. Colgate and R. H. White, ApJ 143, 626 (1966).

[2] R. Buras, H. Janka, M. T. Keil, G. G. Raffelt, and M. Rampp, ApJ 587, 320 (2003).

[3] R. Buras, M. Rampp, H.-T. Janka, and K. Kifonidis, Phys. Rev. Lett. 90, 241101 (2003).

[4] R. Buras, M. Rampp, H.-T. Janka, and K. Kifonidis, A\&A 447, 1049 (2006).

[5] S. W. Bruenn, C. J. Dirk, A. Mezzacappa, J. C. Hayes, J. M. Blondin, W. R. Hix, and O. E. B. Messer, Journal of Physics: Conference Series 46, 393 (2006).

[6] J. Lattimer and F. D. Swesty, Nucl. Phys. A 535, 331 (1991).

[7] S. W. Bruenn, A. Mezzacappa, W. R. Hix, J. M. Blondin, P. Marronetti, O. E. B. Messer, C. J. Dirk, and S. Yoshida, Journal of Physics: Conference Series 180, 012018 (2009).

[8] S. W. Bruenn, ApJS 58, 771 (1985).

[9] S. Reddy, M. Prakash, and J. M. Lattimer, Phys. Rev. D 58, 013009 (1998).

[10] S. Hannestad and G. Raffelt, ApJ 507, 339 (1998).

[11] C. Hanhart, D. R. Phillips, and S. Reddy, Phys. Lett. B 499, 9 (2001).

[12] W. R. Hix and F.-K. Thielemann, ApJ 511, 862 (1999).

[13] F. X. Timmes, R. D. Hoffman, and S. E. Woosley, ApJS 129, 377 (2000).

[14] T. Lee, Ph.D. thesis, University of Tennessee, Knoxville (2008).

[15] A. Heger, N. Langer, and S. E. Woosley, ApJ 528, 368 (2000).

[16] K. N. Yakunin, P. Marronetti, A. Mezzacappa, S. W. Bruenn, C.-T. Lee, M. A. Chertkow, W. R. Hix, J. M. Blondin, E. J. Lentz, O. E. B. Messer, et al., Classical and Quantum Gravity 27, 194005 (2010). 